Methods: A first-person case study analysis of a 2005, Greyhound bus accident, which occurred near Edmonton, Alberta, Canada, was analyzed.

Results: Achieving success and organization of a catastrophic event or natural disaster requires the recognition of the importance of scene control and command, accurate triage and the assurance of destination resource capacity. Multi casualty events are rare, and due to sparse exposure, first responders have limited experience to manage these events effectively. Mass casualty exercises are generally used, although no standardized method exists to evaluate their function and effectiveness. Accurate and timely information are essential in successful multi-casualty events; however, inexperience and limitations often lead to ineffective and inaccurate triage, treatment and transportation of patients.

Conclusion: To ensure efficient and effective mass casualty response, future research should focus on adequate professional development programs for first responders. In addition, tools and instruments to aid in successful multi-casualty events would be an asset in achieving success.

Prehosp Disaster Med 2017;32(Suppl. 1):s234-s235

doi:10.1017/S1049023X1700601X

Comparison of the Predictive Value of Four Burn Scores for Death Risk in Burns Patients in Emergency Departments Hai Hu, Yan Bai

Emergency Department, West-China Hospital, Sichuan University, Chengdu/China

Study/Objective: To evaluate the predictive value of Modified Burn Score (MBS), Abbreviated Burn Severity Index (ABSI), Belgian Outcome in Burn Injury (BOBI), and Injury Severity Score (ISS) for death risk in adult patients with severe thermal burns.

Background: Severe burn patients have high mortality. Accurate prediction of the risk of death in patients with severe burn, contributed to objectively assess the disease, to help clinicians with better clinical decision-making and rational allocation of medical resources. At present, there is a variety of scores on the risk of mortality in burn patients. However there are still, few studies on the prediction of the risk of death in adults with severe thermal burns.

Methods: Retrospectively analyzed data of patients in West China Hospital from 2012 to 2014. The patient's name, gender, age, burn area, and whether complicated with inhalation injury were recorded; and the ISS, MBS, ABSI and BOBI score were calculated. Through drawing Receiver Operating Characteristic curve (ROC curve), the area under the curve of the four scores Area Under Curve (AUC), and the sensitivity and specificity for death prediction were obtained. Based on the sensitivity and specificity for death risk prediction, the Youden index was calculated, the best cutoff value was found, and the best score of death risk prediction for adult patients with severe thermal burn was selected.

Results: There were 85 adult patients with severe thermal burn that were included, with 49 males and 36 females. The AUC of ABSI, MBS, BOBI and ISS were 0.925, 0.825, 0.813, 0.715. Conclusion: ABSI has the best value for the death risk in severe thermal burns adult patients.

Prehosp Disaster Med 2017;32(Suppl. 1):s235

doi:10.1017/S1049023X17006021
New Triage System: Using Digitized Information Entered via a Digital Pen Shinya Yaguchi, Katsubiro Itoh, Hitoshi Yamamura Hirosaki University Hospital, Hirosaki, Aomori/Japan

Study/Objective: We examined the capability of this new triage system, using digitized information entered via a digital pen, as an information tool in times of disaster.

Background: Triage is important in deciding the priority of treatment for many patients injured by disaster. Because the patient's information entered on triage tags and the chronologic list made in each section are done by hand, the process is complicated and accuracy and rapidity cannot be guaranteed. We have created a new triage tag using the Anoto Live Digital Pen (Anoto K.K., Tokyo, Japan).

Methods: The new triage tag uses a check box form as much as possible to reduce readout errors. Furthermore, we developed the system to collect digitized triage information and format the collected data as a chronologic list. We divided the triage data into three categories: check box, numerical characters, and letter characters. We demonstrated this new system during disaster training with simulated patients and assessed whether each category was exactly recognized as digital data.

Results: We were able to collect data from 22 simulated patients. The simulated patient information entered on the handwritten triage tag was quickly digitized, and a chronologic list could be made. Assessment of the accuracy of the digitized data for each category was as follows: check box, $100.0 \%$ (correct number/total number $=127 / 127)$; numerical characters, $71.8 \%(102 / 142)$; and letter characters $51.1 \%(47 / 92)$. The errors in the letter characters were almost exclusively confined to content written in Chinese characters.

Conclusion: This new triage system using digitized information entered via digital pen has some problems with the recognition of letter and numerical characters. However, this system, almost exactly, digitized the data and may be a useful device during times of disaster in the future.

Prehosp Disaster Med 2017;32(Suppl. 1):s235

doi:10.1017/S1049023X17006033

\section{Computer Versus Manual Triage in a Live Disaster}

Simulation

Nisreen Maghraby ${ }^{1}$, Patrick Fok ${ }^{2}$, The Minh Luong ${ }^{2}$, Claudie Bolduc ${ }^{2}$, Valerie Homier ${ }^{3}$

1. Emergency Medicine, Trauma \& Disaster Management, McGill University, Montreal/QC/Canada

2. Emergency Medicine, McGill University Health Center, Montreal/Canada

3. Emergency Medicine, Code Orange Committee, McGill University Health Center, Montreal/QC/Canada

Study/Objective: To compare the use of computerized versus paper-based "Simple Triage and Rapid Treatment" triage in disaster simulations.

Background: Efficient and accurate triage during mass casualty incidents is a critical step of disaster response. Traditionally, triage 
has been manually performed using paper cards, yet new digital technologies claim to be more efficient.

Methods: This prospective observational cross-over study was performed during a live disaster simulation at an urban level 1 trauma center. Healthcare providers (two doctors, two paramedics, and two nurses) each triaged a total of thirty simulated patients, half using paper-based (manual) and half using computer-based (electronic) triage. Speed and accuracy of triage using both methods was measured. Following the exercise, simulated patients and participating health care providers completed a feedback form.

Results: There were no significant differences in triage times (seconds) between manual and electronic methods by doctors $(10.3 \pm 7.2$ vs $15.3 \pm 8.0$, respectively) and nurses $(12.8 \pm 9.8$ vs $11.2 \pm 7.2$ ), whereas the manual method was faster for paramedics $(11.1 \pm 7.2$ vs $21.5 \pm 7.6, p<0.001)$. However, after accounting for extra actions required using the manual method, adjusted triage times for doctors $(21.4 \pm 7.8)$ and nurses $(24.0 \pm 9.9)$ were significantly longer using manual compared to the electronic method ( $\mathrm{p}<0.001)$. Triage accuracy was similar $(\mathrm{p}=0.70)$ between manual $(72 / 90,80 \%)$ and electronic $(75 / 90,83 \%)$. The electronic method was preferred by 4 out of $6(67 \%)$ healthcare providers, while almost half $(14 / 30,47 \%)$ of patients had no preference. While patients commonly perceived the computer method as "less personal" they also perceived it as "better organized". Conclusion: This study suggests that computer triage may be the most efficient triage tool for healthcare providers familiar with the technology. Further studies are required to assess the performance of electronic hospital triage in the context of a rapid patient surge and limited computer availability. We present a framework for assessing the accuracy, efficiency and feasibility of digital technologies in live disaster simulations.

Prehosp Disaster Med 2017;32(Suppl. 1):s235-s236

doi:10.1017/S1049023X17006045

\section{Evaluation of Mass Casualty Triage Algorithms in a Pediatric Population \\ Nitya Reddy, Natalie Lane}

Department Of Emergency Medicine \& Hospitalist Services, Medical College of Georgia at Augusta University, Augusta/GA/United States of America

Study/Objective: This study compared the effectiveness and accuracy of five MCT algorithms in a surrogate pediatric trauma population at a tertiary care children's hospital emergency department.

Background: In disasters, first responders use Mass Casualty Triage (MCT) algorithms to assess victims and direct efforts to provide the greatest good for the greatest number of victims. Several algorithms exist; few were designed for application in pediatric victims.

Methods: An observational, single cohort study with prospective and retrospective data collection was employed. Using a standard observation sheet, prospective data were collected on a convenience sample of pediatric patients with trauma activation levels from one to three, with one being identified as the most severely injured. Trained observers recorded physiologic and treatment observations on injured patients.
An MCT category was determined using each of the five algorithms. After the patient's completed electronic medical record was available, a second reviewer retrospectively determined the patient's MCT category based on a gold standard definition; a standard that uses clinical outcomes to assign a MCT category. The prospective and retrospective categories across the five algorithms were then compared.

Results: The results of this study demonstrate that when existing MCT algorithms are applied to a pediatric trauma population, as if they were disaster victims, they are inconsistent. The algorithms were more accurate for Priority 2 and 3 traumas. JumpSTART, CareFlight, and Triage Sieve assignments were similar and were more accurate than START and SALT. SALT was the least accurate algorithm overall.

Conclusion: A larger sample size is needed to potentially capture a more injured population and a greater variety of patients. Additional research is needed to increase the number of major traumas included, and to increase the sample size overall. The results of this study demonstrate a potential deficit in the algorithm's effectiveness of categorizing pediatric patients in a mass-casualty event.

Prehosp Disaster Med 2017;32(Suppl. 1):s236

doi:10.1017/S1049023X17006057

\section{Trilogie Pilot Study - Assessing the Efficacy of a Triage \\ Sieve Educational Intervention using Non-medical Emergency Service Providers \\ Glen Cuttance \\ Paramedic Unit, Flinders University, Adelaide/SA/Australia}

Study/Objective: The key objective of this pilot study was to determine if a standardised educational intervention would provide non-medical emergency service personnel with enough knowledge to accurately complete a triage sieve questionnaire. A secondary objective was to assess the suitability of a previously utilized triage sieve questionnaire for use with non-medical emergency services.

Background: Non-medical emergency services may be first on scene of a Mass-Casualty Incident (MCI); however, they are not currently trained to undertake primary triage (triage sieve). Methods: Non-medical participants from the Country Fire Service were recruited for this study. All participants completed a triage sieve questionnaire prior to receiving the same standardised educational intervention. Participants were then divided into two groups to repeat the triage sieve questionnaire. One group was provided with an aide-memoire currently used by SA Ambulance Service while the other group received no decision making assistance.

Results: Current accepted triage accuracy rates are $5 \%$ under- and $50 \%$ over-triage. Pre-educational intervention results showed accuracy rates of $65.8 \%$ for under-triage and $50.7 \%$ for over-triage. Post-educational intervention achieved accuracy rates of $2.0 \%$ for under-triage (using an aide-memoir) and 9.2\% (without an aide-memoir); conversely, the group without an aide-memoir achieved a lower over-triage accuracy accuracy rate than those who used an aide-memoir (8.4\% versus $9.5 \%$, respectively). As the improvement in under-triage rate from this study was similar, 